\title{
Aprendizaje dialógico y educación física: principios metodológicos, competencias clave y evidencias científicas
}

José Antonio Andrés-Fabra ${ }^{1}$ y Roberto Ferriz Morell2 ${ }^{*}$

1 CEIP El Castell

2 Universidad CEU Cardenal Herrera

Email: ${ }^{2 *}$ roberto.ferriz@uchceu.es

RESUMEN: Los cambios sociales que se han venido produciendo en los últimos años han hecho necesario adaptar y transformar los procesos de enseñanza y aprendizaje, haciendo evolucionar también el área de educación física (EF). El aprendizaje dialógico (AD) ha sido reconocido como una de las metodologías científicas que contribuyen a fomentar la conexión entre los agentes sociales que pueden intervenir en el contexto educativo (alumnado, familias, docentes, instituciones públicas, voluntariado, etc.). Por extensión, este paradigma podría ser considerado entre las metodologías activas que contribuyen, en el área de EF, a la adquisición de las competencias clave (que deben ser adquiridas para alcanzar un pleno desarrollo personal, social y profesional), conectándolas con las necesidades inherentes a los cambios sociales. Este artículo fue elaborado con el fin de presentar la conexión entre el área de EF y los principios del AD, establecer una relación entre las competencias clave y los principios del AD en el área de $E F$, y presentar evidencias científicas respecto al $A D$ como método para el área de EF. A modo de conclusión, a partir de las evidencias actuales se incluyen recomendaciones orientadas al trabajo de los principios del $A D$ en las clases de EF, así como líneas futuras de investigación a partir de las investigaciones previas realizadas en el área de EF.

PALABRAS CLAVE: Contexto educativo, Comunidades de aprendizaje, Metodología activa, Actuaciones de éxito, Aprendizaje dialógico.

\section{Dialogic learning and physical education: methodological principles, key competencies and scientific evidence}

ABSTRACT: The social changes that have been occurring in recent years have made it necessary to adapt and transform teaching and learning processes. The area of physical education (PE) has also evolved. Dialogic learning (DL) has been recognized as one of the scientific methodologies that contribute to fostering the connection between the social agents that can intervene in the 
educational context (students, families, teachers, public institutions, volunteers, etc.). By extension, this paradigm could be considered among the active methodologies that contribute, in the area of $\mathrm{PE}$, to the acquisition of key competences (which must be acquired to achieve full personal, social and professional development), connecting them with the inherent needs of social changes. This article has been developed in order to: a) present the connection between the area of PE and the principles of $D L, b$ ) establish $a$ relationship between the key competences and DL principles in the PE area, and c) present scientific evidence with respect to DL as a method for the PE area. In conclusion, based on current evidence, recommendations are included as to the use of DL principles in PE classes, as well as indication of future lines of research based on previous investigations carried out in the area of PE.

KEY WORDS: Educational context, Learning communities, Active methodology, Successful actions, Dialogic learning.

\section{INTRODUCCIÓN}

Las necesidades educativas que tiene el alumnado se encuentran en constante cambio. Esta evolución hace que desde el contexto educativo se revise la forma en la que la escuela y sociedad están en contacto. Entre las recomendaciones ofrecidas por la Comisión Europea en el año 2017 en materia de educación para la ciudadanía, se enfatiza en el aprendizaje que incide en el saber hacer (activo), uso del discurso y el debate (interactuar), incidir en los problemas de la vida real del alumnado (relevante), alentar el pensamiento autónomo (crítico), hacer uso del trabajo en grupo y cooperativo (colaborativo) y dar al alumnado voz en su propio aprendizaje (participativo). En este sentido, habilidades como saber expresarse, respetar las ideas opuestas, dialogar para tomar decisiones de forma consensuada, respetar las normas establecidas socialmente o enriquecerse de otras culturas, parecen clave en una sociedad cada vez más universal. Para dar respuesta a la demanda de saber vivir en sociedad, las ciencias de la educación disponen de una corriente educativa que apoya sus principios metodológicos en el estudio de las interacciones sociales y la participación de la comunidad. Entre la teorías científicas que estudian las relaciones interpersonales con un fin educativo se encuentra el aprendizaje dialógico (AD) (Aubert, Flecha, García, Flecha, y Racionero, 2008; Flecha, 1997; Racionero, Ortega, García, y Flecha, 2012). Las consecuencias de la aplicación de esta metodología en el contexto educativo han sido investigadas desde diferentes áreas, incluyendo la educación física (EF) (Castro, 2015).

En línea con la nueva corriente educativa científica, para el área de EF sería recomendable orientar las prácticas docentes en función de los métodos de los que se conoce su éxito para alcanzar las metas propuestas, como es el caso del AD. Toda metodología activa que genere un aprendizaje competencial, como el $A D$, debería contribuir a compartir y construir el conocimiento a través del intercambio verbal y colectivo de ideas (ver Orden ECD/65/2015). Profundizando sobre el AD como método activo para el área de EF, este artículo tuvo tres objetivos (asociados a tres preguntas que podría hacerse el profesorado de EF a la hora de incluir el AD como método para trabajar competencialmente). Primero, presentar la conexión entre el área de EF y los principios del AD. Segundo, establecer una relación entre las competencias clave 
(como elementos sobre los que giran los currículos educativos) y los principios del AD en el área de EF. Tercero, presentar evidencias científicas sobre el AD como método para el área de EF. Estos objetivos abordarían, respectivamente, tres interrogantes; ¿podría trabajar en EF a través del $A D$ ?, ¿qué conexión guardaría el $A D$ con la adquisición de las competencias clave en EF?, ¿existen evidencias científicas que sustenten el uso del AD como método en EF?

\section{DESARROLLO DEL TRABAJO}

\subsection{Principios del aprendizaje dialógico y su conexión con el área de educación física}

El AD (Aubert et al., 2008; Flecha, 1997; Racionero et al., 2012) se apoya en la interiorización del conocimiento desde el plano intersubjetivo y social del individuo, desde el cual se define la construcción personal (Bruner, 1988; Habermas, 1990; Vygotsky, 2000). Por medio de este modelo teórico, se argumenta la necesidad de generar espacios de interacción a través de una comunicación igualitaria entre los distintos agentes educativos, para que, como sugieren Racionero y Padrós (2010), el discente "alcance un nivel de aprendizaje elevado y se embarque en procesos de transformación personal y social" (p.151). Desde esta condición, la perspectiva dialógica atiende a siete principios, constituyéndose como un recurso que permitiría reflexionar acerca de la concreción de la práctica educativa. Desde el área de EF, del mismo modo que sucede con el resto de áreas, se hace necesario reflexionar acerca de cómo construir un AD desde su especificidad para la promoción de un estilo de vida saludable y la adopción de actitudes positivas, conectando contexto educativo y de ocio. Para abordar tal reto, parece interesante incidir en la relación entre los siete principios del AD (Aubert, García y Racionero, 2009; Flecha, 1997) y el área de EF (ver Figura 1).

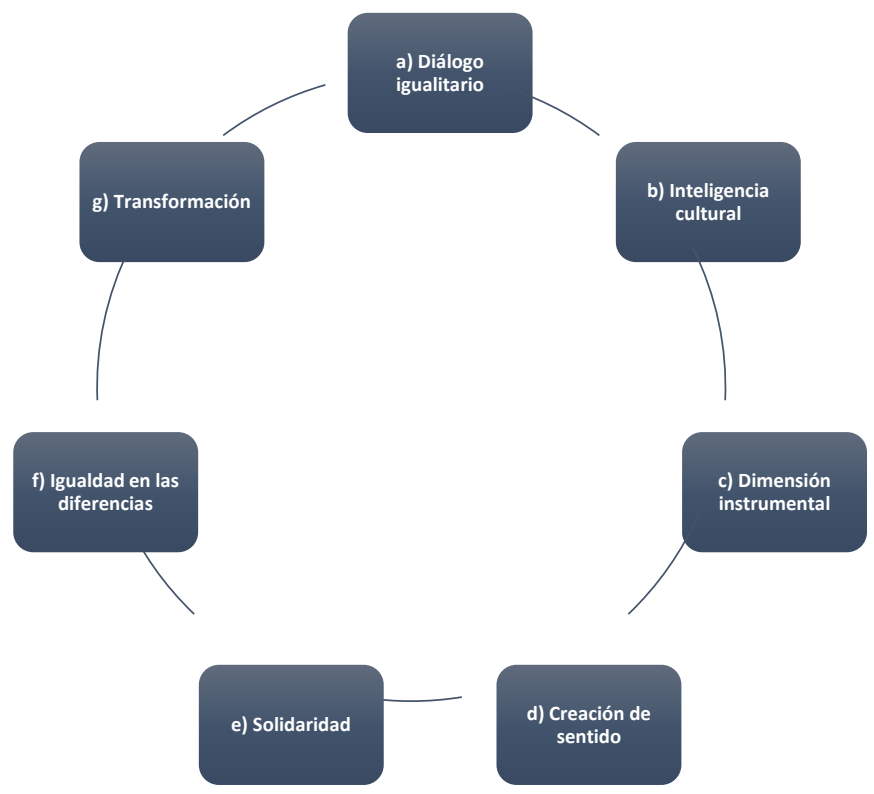

Figura 1. Principios del aprendizaje dialógico

(a) Dialogo igualitario. Se asocia al consenso que determina la validez de los argumentos de las persones participantes en una interlocución. Estas contribuciones 
se aceptan atendiendo al criterio de las mismas según el tema abordado, sin importar el estatus de las personas que los emiten (argumentos de poder o jerarquías sociales) (Elboj, Puigdellívol, Soler y Valls 2002). Por lo tanto, siguiendo este principio no se tiene mayor consideración a las razones de una persona por ser quien es, sino por el sentido común de lo que dice (Castro, 2015). En EF se manifestaría en el fomento del diálogo entre los diferentes agentes sociales participantes en la acción educativa para decidir cuestiones relacionadas con la organización de las sesiones, la toma de decisiones sobre las tareas planteadas, y la prevención y resolución de conflictos (según http://utopiadream.info/ca/centros-en-funcionamiento/experiencias-enca/educacion-fisica-en-ca/, 2010; 2017, diciembre 21). Al inicio de un proceso de enseñanza-aprendizaje fundamentado en el $A D$, sería recomendable regular la tendencia de una participación excesiva por parte de algunos estudiantes, mientras que por otro lado, sería necesario incentivar una mayor intervención de aquellos que manifiestan actitudes inhibidas, con el fin de equilibrar progresivamente un diálogo igualitario en el grupo.

(b) Inteligencia cultural. Todas las personas poseen la capacidad de comunicarse y de actuar en consonancia (Habermas, 1987). Sin embargo, la participación en los distintos contextos sociales puede distar en función de las relaciones sociales previas y de la influencia de los significativos sociales con los que se puede interactuar en el entorno próximo. Estos factores modelan el desarrollo del individuo, posibilitando que el grado de inteligencias culturales adquiridas supongan una interacción exitosa en un determinado ambiente, o en cambio una persona se encuentre en desigualdad en otro contexto (Castro, 2015). El concepto de inteligencia cultural en el AD incluye y valora por igual tres tipos de inteligencias, la inteligencia académica (conocimientos curriculares), la inteligencia práctica (adquisición de habilidades y destrezas) y la inteligencia comunicativa (competencia en comunicación lingüística) (Aubert et al., 2008), tratando de minimizar el riesgo de exclusión asociado al déficit en alguna de ellas. La atención a este principio en EF se daría en la posibilidad que se ofrece a los estudiantes de participar y realizar aportaciones al proceso de enseñanza-aprendizaje a partir de sus habilidades (e.g., comunicativas o motrices), complementando y mejorando las habilidades de los demás mediante el modelado de roles (e.g., feedback positivo del docente o una alumna hacia una actuación de un alumno en la creación y diseño de un circuito de habilidades motrices, que pese a que el alumno no muestra una buena habilidad motriz, sí manifiesta competencia en la toma decisional sobre la organización del material y el tipo de desplazamiento a partir de la inteligencia comunicativa y académica).

(c) Dimensión instrumental. Un enfoque educativo dialógico considera fundamental el aprendizaje de los conocimientos necesarios para vivir en sociedad (Aubert et al., 2009). Esta dimensión aboga por generar contextos dialógicos que contribuyan al aprendizaje curricular de forma específica e interdisciplinar. De esta manera, se multiplica la dimensión instrumental, posibilitando que se alcancen aprendizajes desde los más humanistas a los más técnicos (Elboj et al., 2002). El cumplimiento de dicho principio en EF se sustentaría en un trabajo que integre contenidos propios del área, el componente socio-emocional y el componente lúdico de la práctica en EF. En sus sesiones de EF, el profesorado, además de centrar su atención en el tiempo de compromiso motor, también debería de hacerlo en la promoción de la práctica físico-deportiva de forma autónoma en el tiempo de ocio, como estrategia asociada a la promoción de un estilo de vida activo. En este sentido, existe la necesidad de una coordinación entre el profesorado de EF y los agentes sociales (e.g., familia, monitores, entrenadores, etc.) que pueden influenciar, por ejemplo, en las actividades extraescolares vinculadas a la promoción de la actividad física (Manrique, López, Monjas, Barba, y Gea, 2011). 
(d) Creación de sentido. Se refiere al sentido que los estudiantes confieren a lo aprendido cuando perciben que realmente es útil y aplicable en otros contextos sociales. La forma en que el discente afronta su proceso de formación también lo dotará de sentido. Así, asumir la responsabilidad del aprendizaje propio y del de los demás se constituye como una orientación metodológica relevante, estableciendo al mismo tiempo un nexo entre las estrategias de interacción y resolución de problemas utilizadas en los distintos escenarios sociales en los que se participa (e.g., contexto educativo, relación familiar o actividades extraescolares). También sería importante conectar la creación de sentido con el contexto familiar, haciendo partícipes a las familias en la acción educativa. Para trabajar este principio, en EF se puede promover la creación de proyectos en los que el diálogo y la cooperación tengan un peso importante, buscando la transferencia de competencias específicas del área a la vida cotidiana de los estudiantes (e.g., grupos de debate sobre las implicaciones positivas que tiene para la salud adoptar una dieta saludable o los problemas asociados con una conducta sedentaria) (ver González-Cutre, et al., 2014).

(e) Solidaridad. La educación debe tener un carácter inclusivo, democrático y dirigido al desarrollo de procesos de aprendizajes compartidos. En EF, este principio se concretaría estimulando la empatía, inclusión, y concienciación del cuidado, ayuda y cooperación entre iguales, abordando situaciones de exclusión social. Este enfoque educativo se apoya en el alumnado más hábil o que más sabe, para que ayude y colabore con el resto en pro de un objetivo común (Castro et al., 2014). En EF se puede contribuir a este principio por medio de una agrupación heterogénea del alumnado (e.g., grupos interactivos) que facilite comportamientos solidarios observables y evaluables por el docente (Capllonch y Figueras, 2012; Castro, 2015), independientemente de la diversidad y de las inteligencias coexistentes en los grupos. Al poner en práctica este tipo de agrupaciones, todos los implicados resultarían beneficiados: por una parte los que ayudan profundizan en el aprendizaje debido a una mayor activación neuronal del cerebro (procesos de meta-cognición; Bueno, 2016), mientras que los demás entenderían mejor las explicaciones desde un lenguaje más cercano. Esta mediación dialógica podría tener relación con la optimización del tiempo al incentivar la consolidación de relaciones socio-afectivas positivas, minimizando los conflictos provocados por comportamientos disruptivos.

(f) Igualdad en las diferencias. Es el igual derecho que los individuos tienen a vivir en su diferencia (e.g., origen cultural, estatus socio-económico, género, habilidades sociales, competencias académicas u orientación sexual) (Racionero y Padrós, 2010), oponiéndose a la igualdad como homogeneización del alumnado. Una acción para romper la homogeneidad en las clases de EF es incluir la diversidad que el docente no puede ofrecer por sí mismo. Esto lo puede hacer a través de la agrupación heterogénea del alumnado e incluir la participación activa de recursos humanos (e.g., familia o voluntariado). Otra propuesta es el diseño de actividades inclusivas que atiendan a las distintas inteligencias culturales, sin dar importancia a la situación de partida individual de los estudiantes (Castro, 2015). Sin embargo, las personas de forma inconsciente podríamos manifestar conductas asociadas a nuestros prejuicios, de ahí que introducir espacios dialógicos colectivos sobre este principio al inicio de la sesión, durante la misma (e.g., feed-back interrogativo) y en la vuelta a la calma (e.g., autoevaluación o coevaluación) puede tener una influencia positiva en el tratamiento de la diversidad.

(g) Transformación. De una forma global, la actuación dialógica busca un cambio del contexto educativo para que conecte con su entorno socio-cultural (Capllonch y Figueras, 2012). Esta transformación se orienta a la compensación educativa de las condiciones socio-económicas y culturales externas que reducen las posibilidades de éxito escolar e inclusión social de algunos estudiantes. De una forma 
más concreta, este principio pretende modelar las señales ambientales en el contexto escolar por medio de una interacción dialógica dirigida a un giro conductual positivo del alumnado. Una práctica que podría derivar en la transformación del contexto educativo desde la EF, es la creación de proyectos educativos que involucre directamente a las familias en la educación del alumnado. Esta propuesta mejoraría y diversificaría la interacción, operando, por ejemplo, en la inclusión del alumnado que presenta dificultades de socialización y/o de aprendizaje o en el tratamiento de problemas de convivencia desapercibidos. Otra propuesta que podría ser interesante en el principio de transformación es la realización de programas educativos vinculados a la formación del alumndo y las familias respecto a los beneficios que reporta realizar actividad física regular, ofreciendo estrategias de intervención dirigidas a fomentar la adherencia a la actividad física.

Los principios del aprendizaje dialógico se complementan con orientaciones pedagógicas generales (Elboj et al., 2002; Elboj, Valls, y Fort, 2000). Primero, destaca la participación activa y coordinada (compartiendo ideas e iniciativas desde su ámbito) de los diferentes agentes sociales que podrían estar involucrados en el proceso educativo. Segundo, centralidad del aprendizaje para que el alumnado desarrolle al máximo sus competencias, de manera que el alumnado permanezca el mayor tiempo posible involucrado en su proceso de enseñanza-aprendizaje (para ello se buscará la mejor forma agrupar al alumnado, optimizar el tiempo de las actividades o generar un clima de aula que fomente el aprendizaje). Tercero, las expectativas positivas y la cooperación referente a que tanto el alumnado como quienes interactúan con él tengan la percepción de que pueden lograr sus objetivos-retos, teniendo la oportunidad de demostrarlo (Flecha, 1997). Cuarto, el concepto de progreso permanente evaluado a través de la identificación de aspectos susceptibles de transformación por medio de la implicación de los agentes sociales participantes o la valoración de las consecuencias positivas fruto de la intervención.

El concepto de $A D$ se materializa en el contexto educativo a través de las comunidades de aprendizaje (proyectos educativos) y las prácticas y experiencias de aprendizaje (actuaciones de éxito educativo). Entre las principales actuaciones de éxito se encuentran (INCLUD-ED Consortium, 2011, 2012): los grupos interactivos, las tertulias dialógicas, la formación de familiares, la participación educativa de la comunidad, la formación dialógica del profesorado y el modelo dialógico de prevención y resolución de conflictos. De las diferentes actuaciones de éxito nombradas, Castro (2015) identifica tres prácticas que por sus características parecen ser las más empleadas para involucrar al área de EF: (1) Los grupos interactivos, que se llevan a cabo mediante una intervención directa de voluntarios en las clases de EF, mientras que el docente programa y supervisa. Concretamente, la organización del alumnado se realiza en pequeños grupos heterogéneos de cuatro o cinco componentes, tutorizados por adultos voluntarios, que han recibido información previa del docente, y que centrarán su labor en favorecer el diálogo, asegurar la comprensión de la actividad, dinamizar, fomentar la inclusión, la cooperación para la resolución de problemas y la participación activa de todos los componentes del grupo. Utilizando un sistema de rotación, cada grupo permanecerá durante un tiempo con un voluntario diferente. (2) La participación educativa de la comunidad en las actividades de aprendizaje del alumnado, ya sea en el contexto educativo como el de ocio. Se trata de que los miembros de la comunidad y familias, se involucren en proyectos educativos que tratan de transformar el centro o el entorno próximo. Las familias tomarían decisiones relacionadas con los aprendizajes de sus hijos e hijas y participan en las actividades de aprendizaje del alumnado, ya sea en el horario escolar como fuera. (3) La prevención y resolución de conflictos, que consiste en la forma en la que se alcanzan consensos por parte de la comunidad educativa acerca de las normas que toda la comunidad ha de cumplir para prevenir y superar conflictos, fundamentándose 
en la superación de las desigualdades y el tratamiento del conflicto por medio del consenso.

\subsection{Relación entre las competencias clave y los principios del aprendizaje dialógico en educación física}

La política educativa europea (Comisión Europea, 2007; Unión Europea, 2006) propone un aprendizaje basado en competencias clave. En la misma línea, el sistema educativo español (ver Ley Orgánica 8/2013; Orden ECD/65/2015; Real Decreto 126/2014; Real Decreto 1105/2014) se sustenta en un desarrollo competencial transversal que tanto para el área de EF, como para el resto de áreas, comprende la adquisición de siete competencias clave (comunicación lingüística, competencia matemática y competencias básicas en ciencia y tecnología, competencia digital, aprender a aprender, competencias sociales y cívicas, sentido de iniciativa y espíritu emprendedor, y conciencia y expresiones culturales) contemplando los elementos del currículo dirigidos al saber, saber hacer y saber ser. Entre las diferentes propuestas pedagógicas que podrían contribuir al aprendizaje competencial, el Ministerio de Educación, Cultura y Deporte (Orden ECD/65/2015) sugiere que las competencias sean trabajadas a través de metodologías activas (e.g., aprendizaje cooperativo, aprendizaje basado en proyectos, aprendizaje servicio o aprendizaje basado en problemas), siempre desde una fundamentación científica que contribuya al éxito escolar (Julián, Ibor, Aibar y Aguareles, 2017). Una propuesta que por su naturaleza podría ajustarse a una acción educativa activa es el AD (Aubert et al., 2008; Flecha, 1997; Elboj et al., 2002).

En España, en los últimos años han surgido un importante número de contribuciones que han abordado la relación entre el área de EF y la adquisición de las competencias clave, incluyendo en algunos casos la implementación de metodologías activas (e.g., Figueras, Capllonch, Bláquez, y Monzonís, 2016; Hortigüela, PérezPueyo, y Fernández-Río, 2017; Lleixà, González-Arévalo y Braz-Vieira, 2016; Monzonís y Capllonch, 2014; Rosa, García, y Pérez, 2017). Respecto al AD, son limitados los estudios (e.g., Figueras, Calvo, y Capllonch, 2014) que han abordado su relación con la adquisición de las competencias clave. No obstante, pese a que el trabajo de las competencias clave por medio del AD estaría supeditado a la propuesta didáctica que se plantee, sí que parece coherente pensar que sus principios metodológicos podrían ser más afines a determinadas competencias clave que se pueden trabajar y adquirir con mayor facilidad en EF. Por ejemplo, partiendo de que la perspectiva dialógica de la educación se concreta en contextos educativos que apuestan por el diálogo, la igualdad o la transformación social, parece lógico pensar que el $A D$ desde el área de EF guardaría una relación más estrecha con la (1) competencia en comunicación lingüística, las (2) competencias sociales y cívicas, la (3) competencia en sentido de iniciativa y espíritu emprendedor o la (4) competencia de aprender a aprender, con respecto a otras competencias clave.

Primero, una de las competencias clave que por sus características parecería más afín a los principios del AD aplicados a EF es la (1) competencia en comunicación lingüística. Uno de los máximos exponentes del $A D$ que se podría trabajar en EF a través de esta competencia es el diálogo igualitario. Este principio que se fundamenta en acciones relacionadas con valorar los argumentos ajenos, expresar opiniones constructivas, alcanzar acuerdos y tomar decisiones en la resolución de problemas, llevaría implícito un componente comunicativo e interactivo innato. Una interacción comunicativa de estas características posibilitaría un aprendizaje mutuo (Wells, 2001), y, por ende, contribuiría al desarrollo de procesos internos de meta-cognición (Elboj y Niemela, 2010) asociados con la dimensión instrumental. En EF, la conexión entre el diálogo igualitario y la competencia en comunicación lingüística podría producirse por 
medio de aprender a expresarse en múltiples situaciones comunicativas (e.g., cooperar al experimentar una estructura de aprendizaje cooperativo específica de EF), escuchar evidenciando atención e interés (e.g., ajustando la respuesta a los requerimientos de las actividades y tareas), tener predisposición positiva al diálogo crítico y constructivo, reconociendo el diálogo como un recursos para saber convivir o interesarse por la relación con otros (e.g., para resolver un juego cooperativo). La adquisición de habilidades comunicativas también residiría en el debate sobre la utilidad y la transferibilidad de las competencias clave que se adquieren en el área de EF (e.g., ¿qué nos posibilita conocer y saber jugar a un deporte alternativo nuevo? ¿hay alguna federación o asociación cerca de la zona a la que podamos afiliarnos a ese deporte alternativo?). Así, por medio de la comunicación se produciría una creación de sentido, que asimismo, se generaría mediante situaciones parecidas a las que se dan en contextos relacionados con la práctica de actividad física (e.g., actividades extraescolares deportivas).

Segundo, a medida que se generan aprendizajes para la adquisición de la competencia en comunicación lingüística, también se desarrollarían las habilidades sociales del alumnado, estando este aprendizaje secundado por dos principios del AD, la solidaridad y la igualdad en las diferencias. La contribución de estos dos principios a la adquisición de las competencias clave parece más relacionado con la (2) competencia social y cívica. Por un lado, el principio de solidaridad se produciría mediante el entendimiento que supone la importancia de ayudar y cuidar a los iguales (y otros agentes sociales). Además, este principio también podría guardar relación con mostrar solidaridad e interés y actuar con el fin de resolver problemas que puedan producirse durante las sesiones de EF. Por otro lado, para trabajar la competencia social y cívica desde la perspectiva de la igualdad en las diferencias en EF, el profesorado podría hacer hincapié en ser tolerantes superando prejuicios sobre la diversidad (e.g., sobre la composición corporal de un alumno, la discapacidad física de una alumna o los estereotipos de género que puedan existir asociados a una determinada actividad físico-deportiva). Además, la (1) competencia en comunicación lingüística (así como las competencias sociales y cívicas), se podrían abordar desde las inteligencias culturales manifestadas en el grupo, sustentándose también en la diversidad; en este caso, la referente al tipo de habilidades predominantes (académicas, comunicativas y/o prácticas). Los postulados sobre la inteligencia cultural ofrecen la posibilidad de enriquecer las interacciones y por consiguiente respaldar la mejora de las habilidades comunicativas y sociales de los educandos. En esta línea argumentativa, el AD podría incidir de forma específica en la (2) competencia social y cívica a través de la interiorización de las normas para participar en EF y en general mediante la comprensión del código de conducta aceptado por la sociedad (e.g., democracia, justicia, ciudadanía y derechos humanos, entre otros). Asimismo, adquirir (2) competencias sociales y cívicas, interesándose por resolver problemas de la sociedad o saber comunicarse con otros grupos sociales, permitiría al alumnado realizar aportaciones a las actividades de la comunidad (e.g., participar en un proyecto de aprendizaje servicio en el que la EF es el motor del proyecto), relacionando así esta competencia con el principio de transformación del AD.

Tercero, por sus características, la (3) competencia de iniciativa y espíritu emprendedor también estaría directamente relacionada con los principios del AD. Algunos de los principios de esta vertiente pedagógica, como es el caso del diálogo igualitario, la inteligencia cultural o la igualdad en las diferencias, de manera intrínseca, podrían contribuir a modelar ambientes en EF dirigidos al apoyo y la valoración constructiva de la intervención discente. De este modo, se podría minimizar la posible presión externa que puede percibir el alumnado al recibir una evaluación negativa de su participación en clase (e.g., al realizar una tarea motriz) y/o aportaciones personales o grupales (e.g., propuestas para practicar nuevos juegos motrices). De 
este modo, se podría mediar en una participación continuada y en una mayor adaptación a las exigencias de la práctica. Esta disposición de los discentes en las clases de EF daría la posibilidad de contribuir a un desarrollo deseado del autoconcepto y la autoestima en pro de una transferencia de las conductas aprendidas a otros contextos sociales. Probablemente uno de los principios del AD que más relación guarda con la (3) competencia que persigue que el alumnado tome la iniciativa respecto a su proceso de formación, es el principio de transformación, cuyo objetivo en este caso sería dotar al alumnado de una competencia que le permita manifestar comportamientos autónomos relacionados a la práctica de actividad física.

Cuarto, la interacción dialógica sugerida en el $A D$ ayudaría a un mayor entendimiento de las reglas heurísticas que afectan a la práctica (CREA; Community of Research on Excellence for All, 2002), pudiendo ayudar a la adquisición de habilidades relacionadas con la (4) competencia de aprender a aprender. En este caso, el fomento de procesos comunicativos e interactivos guiados por un diálogo igualitario y por relaciones sociales sustentadas en los principios dialógicos de solidaridad e igualdad, posibilitaría una predisposición discente hacia la autogestión de aprendizajes y el uso de estrategias dirigidas a la resolución autónoma de problemas-retos. Además, la influencia del $A D$ en la (4) competencia de aprender a aprender se podría ver reforzada con la creación de situaciones de aprendizaje significativo asociadas a contenidos específicos de EF desde el principio de dimensión instrumental. Para contribuir a esta competencia, los educandos pueden aportar, por ejemplo, conocimientos o habilidades, a partir de la interacción desde las distintas inteligencias culturales coexistentes en el grupo, hecho que podría enriquecer el proceso de enseñanza-aprendizaje a favor de un aprendizaje autónomo y compartido. Por su parte, para el principio de creación de sentido, se podrían proponer situaciones parecidas a las realizadas en otros contextos sociales (e.g., ceder al alumnado la responsabilidad de inventar un juego y decidir los diferentes elementos de la práctica como el espacio, materiales, tiempo, normas, etc.). También, desde esta dimensión se podría contribuir a que los estudiantes actúen de forma independiente y espontánea, siendo protagonistas en la planificación, supervisión y auto-evaluación de sus procesos de formación.

Quinto, a pesar de que existen competencias clave que a priori no presentarían una relación directa con los principios del $A D$ en EF, un trabajo interdisciplinar guiado desde el área de EF, permitiría la adquisición del resto de competencias. Es por ello que se podría trabajar desde la perspectiva del área de EF; la competencia matemática, en ciencias y tecnología (e.g., actividades vinculadas al área de matemáticas de alfabetización numérica, cálculo mental, unidades de medida, por ejemplo para diseñar una zona de juego de un deporte alternativo), competencia digital (e.g., proyectos relacionados con las tecnologías del aprendizaje y el conocimiento, por ejemplo, para utilizar una aplicación móvil que recopila la cantidad de actividades físicas que se realizan o el número de pasos), y competencia de conciencia y expresiones culturales (e.g., conocer y valorar los deportes autóctonos, juegos populares o bailes tradicionales).

Ante este planteamiento, en EF a través de la concreción del AD se trataría de responder a las demandas referentes a la adquisición de las competencias clave propuestas por el Ministerio de Educación, Cultura y Deporte (Orden ECD/65/2015). Por lo tanto, el área, tomando como hilo conductor el desarrollo competencial, podría generar un vínculo con otros contextos sociales, alcanzando un mayor estatus y reconocimiento como espacio educativo en el que los estudiantes aprendan a saber, saber hacer y saber ser en sociedad. 


\subsection{Evidencia científica y aprendizaje dialógico en educación física}

Las evidencias científicas de las que se disponen respecto a la aplicación del $A D$ como método en el área de EF parecen limitadas (Castro, 2015). Gran parte del cuerpo científico sobre AD en EF ha sido fruto del proyecto Juega, Dialoga y Resuelve. La superación de conflictos en Educación Física mediante el modelo comunitario. Diseño de un programa específico para Comunidades de Aprendizaje (Capllonch, 2008-2011). No obstante, aunque la investigación sobre AD en EF es relativamente reciente, sí existe una sólida base científica que apoya su aplicación al contexto educativo en general (e.g., ver Aubert, Molina, Shubert, y Vidu, 2017; García, Mircea, y Duque, 2010; Gatt, Ojala, y Soler, 2010) y de forma exclusiva en algunas áreas, como por ejemplo, en matemáticas (e.g., ver García-Carrión y Díez-Palomar, 2015; OrdóñezSierra, Rodríguez-Gallego, y Rodríguez-Santero, 2017). Además, cabe destacar que la mayoría de los estudios realizados sobre el AD en EF han sido abordados bajo la metodología comunicativa crítica (Gómez, Latorre, Flecha, y Sánchez, 2006), que se apoya en la metodología cuantitativa y cualitativa, tratando de generar nuevo conocimiento a partir de la subjetividad y la reflexión conjunta de todos los agentes sociales relacionados con la investigación.

Gran parte de los estudios empíricos en el área de EF desde una perspectiva dialógica han sido realizados bajo actuaciones de éxito asociadas a un modelo dialógico de prevención y resolución de conflictos (Buscà, Ruiz, y Rekalde, 2014; Figueras, Calvo y Capllonch, 2014; Martín y Ríos, 2014; Monzonís y Capllonch, 2014) y a través de metodología cualitativa. En este sentido, una revisión de la literatura realizada por Capllonch, Figueras y Lleixà (2014), relacionada con prevención y resolución de conflictos, sugiere que intervenir desde las escuelas transformadas en comunidades de aprendizaje y utilizar la práctica físico-deportiva (involucrar a la EF) como recurso de integración puede ser una estrategia de intervención idónea para fomentar la cohesión entre los agentes de la comunidad.

Buscà et al. (2014) realizaron un diseño no experimental-descriptivo con el fin de analizar la influencia que la comunidad de aprendizaje tuvo sobre la prevención y resolución de conflictos en las actividades físico-deportivas que se desarrollaban en la escuela. En este estudio participaron seis comunidades de aprendizaje (276 participantes entre profesorado, familias, voluntariado y alumnado) y se desarrollaron seis relatos comunicativos de vida, empleando para el estudio una metodología comunicativa. Entre los resultados obtenidos destaca que profesorado, familias y voluntariado valoraron el diálogo, aplicando el modelo comunitario, como el camino más apropiado para la prevención y resolución de conflictos, tanto en las clases de EF como en el contexto educativo en general. De forma complementaria al estudio de Busà et al. (2016), Figueras, et al. (2014) focalizaron la atención en profundizar en la parte cualitativa del proyecto de investigación, realizando 12 entrevistas, seis a cada uno de los grupos de miembros adultos de las comunidades de aprendizaje (profesorado, familiares y voluntariado). Con el estudio se consultó acerca de opinión, creencia, y valoración que los adultos de las comunidades de aprendizaje tenían sobre las causas que provocaban los conflictos que se daban en EF y las formas de darles respuesta. Entre las principales conclusiones que extraen los autores de su estudio se identifican dos grandes ideas; a) las barreras que dificultarían el éxito de un modelo comunitario (posible falta de igualdad de oportunidades entre alumnado para lograr el éxito en EF, la posible influencia negativa que tendrían los medios de comunicación, la prevalencia de un modelo autoritario para la resolución de conflictos, la posible falta de competencias comunicativas y recursos de los agentes de la comunidad para el diálogo, y la normalización del conflicto por parte de los adultos) y b) generar recursos que favorezcan la resolución comunitaria de los conflictos en EF (llegar a consensos sobre el funcionamiento del área y las normas para la prevención y resolución de los 
conflictos, orientar la EF hacia la adquisición de valores a través de metodologías participativas, y utilizar la competición como herramienta educativa para sacar partido sus posibilidades como herramienta para vivenciar la gestión del conflicto). Por su parte, Martín y Ríos (2014), como parte del mismo proyecto que los autores anteriores (Busà et al., 2016; Figueras et al., 2014) se centraron en la opinión del alumnado que participó en las comunidades de aprendizaje en relación a la prevención y resolución de conflictos en EF. En este estudio participaron 184 estudiantes y se profundizó en seis relatos de vida de alumnado de quinto y sexto de la etapa de educación primaria obligatoria. Este trabajo destaca que el alumnado cree en la necesidad de hacer uso del diálogo con el profesorado, el voluntariado y las familias para crear, poner en práctica y sancionar, cuando sea necesario, los comportamientos y normas de convivencia en la comunidad y en las clases de EF.

Respecto a la tipología de estudios de intervención, Monzonís y Capllonch (2014) realizaron un estudio transversal (cuantitativo y cualitativo) con el fin de realizar un cambio en el clima de convivencia en un centro educativo de educación primaria. Por medio del trabajo de la competencia social y ciudadana se trató de abordar la prevención y resolución de conflictos desde el área de EF. Tras un pre-test, se diseñaron estrategias de intervención que se pusieron en práctica en las tutorías (26 sesiones) y en las clases de EF (54 sesiones), con una duración de una hora por sesión. Aunque, en el grupo de sexto de primaria únicamente se aplicó la intervención que correspondía a EF (entre las estrategias de intervención los autores destacaron la utilización; del razonamiento moral a través de historias hipotéticas que contraponen valores, los debates breves de concienciación de los valores tratados, la elaboración de carteles resumen de los acuerdos consensuados democráticamente por el alumnado, el aprendizaje entre iguales, la autoevaluación de cada sesión y el respeto del ritmo de aprendizaje). Concretamente, para las sesiones de EF se empleó el modelo de Hellison (1995) como estrategia de intervención. Tras el estudio, las respuestas que más se dieron tras los conflictos fueron ignorar el hecho que causó el conflicto o emplear el diálogo para darle solución. Además, se redujeron la resolución de conflictos que implicaban violencia, se dieron más mediaciones con ayuda de compañeros y el diálogo autónomo. En general, los resultado del estudio evidenciaron una mejora de los procesos relacionales, destacando los autores el trabajo cooperativo y el aprendizaje entre iguales como recursos metodológico para desarrollar las habilidades comunicativas del alumnado en EF.

Otra de las actuaciones de éxito sobre las que se ha investigado en EF ha sido la aplicación de grupos interactivos (Capllonch y Figueras, 2012; Castro, Gómez, y Macazaga, 2014; Rivera, Moreno, y Giles, 2017). Capllonch y Figueras (2012) realizaron un estudio cuantitativo en el que participaron seis comunidades de aprendizaje y alumnado de quinto y sexto de la educación primaria obligatoria. Se consultó acerca de la opinión del alumnado acerca del funcionamiento de las clases de EF con el fin de comprobar la medida en la que se estaban aplicando los principios del $A D$ en relación a el clima de aula, las estrategias aplicadas, las formas de organización, la manera de trabajar los contenidos y la orientación de las actividades, el nivel de responsabilidad del alumnado respecto a su aprendizaje, la utilización del diálogo y las expectativas de éxito de los aprendizajes. Los autores concluyen que las comunidades de aprendizaje en las que participa el área de EF: a) se propiciar un clima de respeto entre los participantes, b) se fomentar el trabajo cooperativo en grupos heterogéneos, c) se promover la responsabilidad entre el alumnado, c) se fomenta el diálogo, y d) se generan expectativas de éxito respecto a los aprendizajes independientemente del nivel de partida. En línea con otros estudios previos, también Castro et al. (2014) sugirieron que con la aplicación de los grupos interactivos en EF se propicia la creación de un clima de diálogo igualitario (aprender el principio de diálogo igualitario, destacando el esfuerzo docente por adoptar una actitud empática, 
sincera y honesta centrada en los argumentos y no en la posición o rol que ocupan las personas que emiten las ideas), el éxito educativo (relacionado con la dimensión instrumental que ayuda al alumnado a responder a las demandas sociales asociadas con la toma de decisiones, ser creativos, esforzarse y el respeto por las normas y los demás) y se propicia una educación inclusiva (vivenciar el principio de solidaridad, de forma que el alumnado que destaca en el saber, saber hacer ayuda y trabaja junto con los que saben menos o no son tan habilidosos como ellos en una actividad). Los autores destacan la necesidad de poner en práctica actividades de tipo cooperativo a través de los grupos interactivos, con el fin de fomentar el éxito grupal y trabajar el principio de solidaridad del AD.

Uno de los estudios más recientes en los que se ha trabajado con grupos interactivos en EF es el de Rivera et al. (2017). Además de los grupos interactivos, los autores utilizaron la metodología de aprendizaje servicio y las tertulias dialógicas (construcción colectiva de significado y conocimiento en base al diálogo por parte del alumnado participante en una tertulia) en la etapa de educación primaria obligatoria (desde tercer a quinto curso). En el estudio, con el rol de voluntarios participaron estudiantes de grado de educación primaria, que intervinieron en siete comunidades de aprendizaje. Aunque, como afirman los autores, se trata de un estudio transversal preliminar, entre las conclusiones se destaca la satisfacción de los agentes participantes en el proceso (estudiante universitario y profesorado de educación primaria) al contar con los grupos interactivos en sus clases.

\section{CONCLUSIONES}

Sustentado en el AD como una metodología científica, este trabajo tuvo tres objetivos. En primer lugar, presentar la relación entre la EF y los principios del AD. En segundo lugar, establecer una relación entre las competencias clave y los principios del AD en EF. En tercer lugar, mostrar evidencias científicas sobre el AD como método para EF. Incluir en el área de EF el AD como metodología activa que conecte el contexto educativo con la sociedad, respondería a las necesidades demandadas por la sociedad para transformar la comunidad educativa (Capllonch y Figueras, 2012; Molina, 2007). En los últimos años, diferentes estratos de la población se han encontrado en desventaja en materia educativa debido al entorno socio-cultural y económico al que pertenecían (Soler y Acosta, 2005). Ante esta situación social, según Elboj et al. (2002), el AD tiene como uno de sus principales objetivos ofrecer desde el contexto educativo la igualdad de oportunidades en la adquisición de competencias y el acceso "desde su propia situación, al máximo de posibilidades culturales y educativas, de manera que sus aprendizajes puedan ser iguales 0 superiores" (p. 75). Esta idea de la educación, aúna el contexto educativo y el contexto social del alumnado, interviniendo de forma global y coordinada desde ambos contextos (Elboj et al., 2000). Fruto de este trabajo se pueden extraer dos conclusiones principales, por un lado respecto a las recomendaciones metodológicas que surgen de la investigación sobre $A D$ en $E F$, y por otro lado, en cuanto a las posibles futuras líneas de trabajo en esta perspectiva educativa.

A modo de resumen, a partir de las evidencias y sugerencias que se recogen en la bibliografía específica se incluyen recomendaciones prácticas que estarían orientadas al trabajo de los principios del AD en las clases de EF: 
1) Diálogo igualitario: al interactuar en el desarrollo de una actividad (e.g., solucionar un reto cooperativo), los participantes debería de hacerlo desde el diálogo centrado en los argumentos y no en la posición o figuras de autoridad que ocupan.

2) Inteligencia cultural: en el desempeño de una actividad motriz, trabajar, valorar y evaluar lo concerniente a la competencia académica (conocimientos curriculares), práctica (adquisición de habilidades y destrezas) y comunicativa (competencia en comunicación lingüística).

3) Dimensión instrumental: crear situaciones de aprendizaje de tipo competencial, de forma que lo aprendido en EF tenga transferencia al contexto de ocio del alumnado.

4) Creación de sentido: posibilitar la percepción de que los aprendizajes y competencias adquiridas son útiles en otros marcos sociales. El proceso de creación de sentido se consolida con la participación activa de la familia en situaciones de aprendizajes similares a las desarrolladas en el contexto extraescolar.

5) Solidaridad: apostar por metodologías activas que fomentan la cooperación entre el alumnado, como por ejemplo el aprendizaje cooperativo, que fomentan actitudes positivas (e.g., la inclusión del alumnado con necesidades específicas de apoyo educativo o la ayuda al alumnado menos competente motrizmente en una actividad concreta).

6) Igualdad en las diferencias: fomentar nuevas situaciones de aprendizaje en la que el alumnado tenga que adaptar sus respuestas en función de los agentes con los que interviene. Por ejemplo, variar las agrupaciones, interactuar con diferentes agentes sociales o experimentar nuevos contenidos en los que la percepción de competencia individual y grupal haga que se experimenten nuevos roles (en ocasiones unos alumnos serán más competentes que otros y tendrán que respetar sus diferencias y ayudarse mutuamente para alcanzar un objetivo grupal).

7) Transformación: Incluir la participación del área de EF como parte importante de un proyecto educativo que tiene por fin mejorar el contexto educativo o el entorno.

Además, fruto del presente trabajo se extraen algunas ideas clave que podrían derivar en futuras líneas de investigación para profundizar sobre el $A D$ como método en EF:

1) Ahondar en las aportaciones que desde el área de EF se pueden realizar desde un punto de vista competencial (asociado al saber, saber hacer y saber ser) a la adquisición de un AD (empleando metodología cuantitativa y cualitativa).

2) Son necesarios estudios de intervención (de tipo transversal y longitudinal) en los que el área de EF sea incluida dentro de un proyecto educativo de una comunidad de aprendizaje, así como estudios en los que se analicen las consecuencias positivas y/o negativas de la aplicación de actuaciones de éxito como metodología sobre la que gire una unidad didáctica en EF.

3) Profundizar acerca del tipo de actuaciones de éxito que resultarían más eficientes a la hora de su puesta en práctica en el área de EF.

4) Analizar y comparar la efectividad del $A D$ en diferentes etapas educativas (la mayoría de la investigación hasta la fecha se centra en la etapa de educación primaria obligatoria) en las que la EF forma parte de currículo educativo: educación primaria obligatoria, educación secundaria obligatoria y educación secundaria postobligatoria.

5) Diseñar y analizar estudios de intervención que incluyan la formación del profesorado de EF en el AD. Se podría realizar un proceso de formación dirigido a conocer las posibilidades que ofrece el AD como método competencial en EF, 
para posteriormente dotar al profesorado de estrategias de intervención específicas para el área de EF.

6) Creación y validación de instrumentos para medir los principios metodológicos y las estrategias de intervención específicas del $A D$ (para el ámbito educativo en general y para el área de EF de forma específica). Algunos de estos instrumentos también podrían ser específicos para los diferentes agentes sociales que intervienen en el contexto educativo (e.g., dirigidos a medir la percepción del alumnado acerca del apoyo que voluntarios o familiares ofrece para que se genere un diálogo igualitario). Disponer de este tipo de instrumentos ayudaría a comparar los resultados obtenidos en los diferentes estudios, posibilitando realizar futuros trabajos de revisión y meta-análisis que contribuyan a transferir lo investigado a la comunidad educativa. Además, disponer de estas herramientas permitiría medir el constructo (AD como medida unidimensional) o dimensiones del AD (una para cada principio metodológico) para ser relacionado con otras variables que puedan ser identificadas como antecedentes 0 consecuencias del AD y explicar así el mayor porcentaje de varianza sobre consecuencias positivas y/o negativas.

A modo de conclusión, en general, como sugieren algunos investigadores (e.g., Capllonch y Figueras, 2012; Castro, 2015), todavía son necesarias más evidencias científicas que ofrezcan recursos a los docentes del área acerca de cómo aplicar el AD de forma específica en EF. No obstante, sí se pueden extraer algunas sugerencias clave que se han presentado en el presente trabajo y que podrían ser de utilidad para los docentes desde una vertiente práctica y para los investigadores en el análisis de este paradigma educativo.

\section{REFERENCIAS}

Aubert, A., Flecha, A., García, C., Flecha, R., y Racionero, S. (2008). Aprendizaje dialógico en la sociedad de la información. Barcelona. Hipatia.

Aubert, A., García, C., y Racionero, S. (2009). El aprendizaje dialógico. Cultura y Educación, 21(2),129-139. doi: http://dx.doi.org/10.1174/113564009788345826

Aubert, A., Molina, S., Schubert, T., y Vidu, A. (2017). Learning and inclusivity via Interactive Groups in early childhood education and care in the Hope school, Spain. Learning, Culture and Social Interaction, 13, 90-103. doi: https://doi.org/10.1016/j.Icsi.2017.03.002

Buscà, F., Ruiz, L., y Rekalde, I., (2014). Tratamiento del conflicto en las Comunidades de Aprendizaje a través de la educación física. Retos. Nuevas tendencias en Educación Física, Deporte y Recreación, 25, 156-161.

Bruner, J. S. (1988). Desarrollo cognitivo y educación. Madrid: Morata.

Capllonch, M. (2017, diciembre 21). Juega Dialoga y Resuelve. La superación de conflictos en educación física mediante el modelo comunitario. Diseño de un programa específico para comunidades de aprendizaje, con referencia: SEJ2007-61757/EDUC. Plan Nacional I+D+I (2007-2009). Recuperado de http://utopiadream.info/ca/centros-en-funcionamiento/experiencias-enca/educacion-fisica-en-cal 
Capllonch, M., y Figueras, S. (2012). Educación física y comunidades de aprendizaje. Estudios Pedagógicos, 38(número especial), 231-247. doi: http://dx.doi.org/10.4067/S0718-07052012000400013

Capllonch, M., Figueras, S., y Lleixà, T. (2014). Prevención y resolución de conflictos en educación física: estado de la cuestión. Retos, 25, 149-155.

Castro, M., Gómez, A., y Macazaga, A. M. (2014). Aprendizaje dialógico y grupos interactivos en educación física. Retos, 25, 174-179.

Castro, M. (2015). Aprendizaje dialógico y educación física: Hacia una educación física y un deporte escolar promotores de salud, éxito escolar y cohesión social. Omnia Science, 11(3), 393-417. doi: http://dx.doi.org/10.3926/ic.636

Comisión Europea. (2007). Competencias clave para un aprendizaje permanente. Un marco de referencia europeo. Luxemburgo: Oficina de Publicaciones de la Unión Europea.

Comisión Europea. (2017). Citizenship education at school in Europe. Luxemburgo: Oficina de Publicaciones de la Unión Europea.

Community of Research on Excellence for All (CREA). (2002). Comunidades de aprendizaje. Barcelona: Universidad de Barcelona.

Elboj, C., y Niemela, R. (2010). Sub-communities of mutual learners in the classroom: the case of interactive groups. Revista de Psicodidáctica, 15(2), 177-189.

Elboj, C., Puigdellívol, I., Soler, M., y Valls, R. (2002). Comunidades de aprendizaje. Transformar la educación. Barcelona. Graó.

Elboj, C., Valls, R., y Fort, M. (2000). Comunidades de aprendizaje. Una práctica educativa para la sociedad de la información. Cultura y Educación, 12(1-2), 129-141.

Figueras, S., Calvo, J., y Capllonch, M. (2014). Prevención y resolución del conflicto en educación física desde la perspectiva de los adultos miembros de la comunidad educativa en las comunidades de aprendizaje. Retos, 25, 168-173.

Figueras, S., Capllonch, M., Bláquez, D., y Monzonís, N. (2016). Competencias básicas y educación física: estudios e investigaciones. Apunts. Educación Física y Deportes, 123, 34-43.

Flecha, R. (1997). Compartiendo palabras. Barcelona: Paidós.

García-Carrión, R., y Díez-Palomar, J. (2015). Learning communities: Pathways for educational success and social transformation through interactive groups in mathematics. European Educational Research Journal, 14(2), 151-166. doi: http://dx.doi.org/10.1177/1474904115571793

García, R., Mircea, T., y Duque, E. (2010). Socio-cultural transformation and the promotion of learning. Revista de Psicodidáctica, 15(2), 207-222. doi: http://dx.doi.org/10.1387/RevPsicodidact.812

Gatt, S., Ojala, M., y Soler, M. (2011). Promoting social inclusion counting with everyone: Learning Communities and INCLUD-ED. International Studies in 
Sociology of Education, 21(1), 33-47. doi: http://dx.doi.org/10.1080/09620214.2011.543851

Gómez, J., Latorre, A., Sánchez, M., y Flecha, R. (2006). Metodología comunicativa crítica. Barcelona: El Roure Ciencia.

González-Cutre, D., Ferriz, R., Beltrán-Carrillo V. J., Andrés-Fabra, J. A., MonteroCarretero, C., Cervelló, E., y Moreno-Murcia, J. A. (2014). Promotion of autonomy for participation in physical activity: a study based on the transcontextual model of motivation. Educational Psychology, 34, 367-384. dio: http://dx.doi.org/10.1080/01443410.2013.817325

Habermas, J. (1987). Teoría de la acción comunicativa: racionalidad de la acción y racionalización social. Madrid: Taurus.

Habermas, J. (1990). El pensamiento postmetafísico. Madrid: Taurus Humanidades.

Hortigüela, D., Pérez-Pueyo, A., y Fernández-Río, J. (2017). Implantación de las competencias: percepciones de directivos y docentes de educación física. Revista Internacional de Medicina y Ciencias de la Actividad Física y del Deporte, 17(66), 261.281. doi: http://dx.doi.org/10.15366/rimcafd2017.66.004

INCLUDE-ED Consortium (2011). Actuaciones de éxito en las escuelas europeas. Madrid: Ministerio de Educación.

INCLUD-ED Consortium. (2012). Final report. strategies for inclusion and social cohesion in Europe from education. Bruselas: Comisión Europea.

Julián, J. A., Ibor, E., Aibar, A., y Aguareles, I. (2017). Educación física, motor de proyectos. Támdem, 56, 6-15.

Ley Orgánica 8/2013, de 9 de diciembre, para la mejora de la calidad educativa. Boletín Oficial del Estado. Madrid, 10 de diciembre de 2013, núm. 295, pp. 97858-97921.

Lleixà, T., González-Arévalo, C., y Braz-Vieira, M. (2016). Integrating key competences in school physical education programmes. European Physical Education Review, 22, 1-20. doi: http://dx.doi.org/10.1177/1356336X15621497

Manrique, J. C., López, V. M., Monjas, R., Barba, J. J., y Gea, J. M. (2011). Implantación de un proyecto de transformación social en Segovia (España): desarrollo de un programa de deporte escolar en toda la ciudad. Apunts. Educación Física y Deportes, 105, 58-66. doi: http://dx.doi.org/10.5672/apunts.2014-0983.es.(2011/3).105.07

Martín, M., y Ríos, O. (2014). Prevención y resolución del conflicto en educación física desde la perspectiva del alumnado. Retos, 25, 162-167.

Molina, S. (2007). Los grupos interactivos: una práctica de las comunidades de aprendizaje para la inclusión del alumnado con discapacidad (Tesis doctoral). Barcelona, Universitat de Barcelona. Recuperado de https://goo.gl/1xdUt1

Monzonís, N., y Capllonch, M. (2014). La educación física en la consecución de la competencia social y ciudadana. Retos, $25,180-185$. 
Orden ECD/65/2015, de 21 de enero, por la que se describen las relaciones entre las competencias, los contenidos y los criterios de evaluación de la educación primaria, la educación secundaria obligatoria y el bachillerato. Boletín Oficial del Estado. Madrid, 29 de enero de 2015, núm. 25, pp. 6986-7003.

Ordóñez-Sierra, R., Rodríguez-Gallego, M., y Rodríguez-Santero, J. (2017). Grupos Interactivos como estrategia para la mejora educativa: estudio de casos en una comunidad de aprendizaje. Revista de Investigación Educativa, 35(1), 71-91. doi: http://dx.doi.org/10.6018/rie.35.1.247061

Unión Europea. (2006). Recomendación del Parlamento Europeo y del Consejo del 18 de diciembre de 2006, sobre las competencias clave para el aprendizaje permanente (2006/962/CE). Recuperado: 5/06/2017 de https://goo.gl/G73ZHU

Racionero, S, Ortega, S, García, R, y Flecha, R. (2012). Aprendiendo contigo. Barcelona: Hipatia.

Racionero, S., y Padrós, M. (2010). The dialogic turn in educational psychologoly. Revista de Psicodidáctica, 15(2), 143-162. doi: http://dx.doi.org/10.1387/RevPsicodidact.808

Real Decreto 126/2014, de 28 de febrero, por el que se establece el currículo básico de Educación Primaria. Boletín Oficial del Estado. Madrid, 1 de marzo de 2014, núm. 52, pp. 19349-19420.

Real Decreto 1105/2014, de 26 de diciembre, por el que se establece el currículo básico de la Educación Secundaria Obligatoria y del Bachillerato. Madrid, 3 de enero de 2015, núm. 3, pp. 169-546.

Rivera, E., Moreno, A., y Giles, J. (2017). Un estudio sobre la mejora educativa a través de los grupos interactivos en una comunidad de aprendizaje. Infancia, $\begin{array}{llll}\text { Educación } y & \text { Aprendizaje, } 3(2), & 524-530 . & \text { doi: }\end{array}$ http://dx.doi.org/10.22370/ieya.2017.3.2.774

Rosa, A., García, E., y Pérez, J. J. (2017). Propuesta práctica para el desarrollo de las competencias clave mediante una metodología basada en los ambientes de aprendizaje. EmásF: revista digital de educación física, 48, 10-26. Recuperado de https://dialnet.unirioja.es/servlet/articulo?codigo=6121662

Soler, M., y Acosta, S. (2005). Comunidades de aprendizaje: propuesta educativa igualitaria en la sociedad de la información. Artículo presentado en Jornadas arte y educación en la sociedad de la información. Barcelona: Fundació La Caixa.

Bueno, D. (2016). Cerebroflexia. Barcelona: Plataforma.

Vygotsky, L. S. (2000). El desarrollo de los procesos psicológicos superiores. Barcelona: Crítica.

Wells, G. (2001). Indagación dialógica. Barcelona: Paidós. 